\title{
Attention effects during visual short-term memory maintenance: Protection or prioritization?
}

\author{
Michi Matsukura \\ University of Iowa, Iowa City, Iowa \\ STEven J. LuCK \\ University of California, Davis, California \\ AND \\ Shaun P. Vecera \\ University of Iowa, Iowa City, Iowa
}

\begin{abstract}
Interactions between visual attention and visual short-term memory (VSTM) play a central role in cognitive processing. For example, attention can assist in selectively encoding items into visual memory. Attention appears to be able to influence items already stored in visual memory, as well; cues that appear long after the presentation of an array of objects can affect memory for those objects (Griffin \& Nobre, 2003). In five experiments, we distinguished two possible mechanisms for the effects of cues on items currently stored in VSTM. A protection account proposes that attention protects the cued item from becoming degraded during the retention interval. By contrast, a prioritization account suggests that attention increases a cued item's priority during the comparison process that occurs when memory is tested. The results of the experiments were consistent with the first of these possibilities, suggesting that attention can serve to protect VSTM representations while they are being maintained.
\end{abstract}

The goal of the present study was to examine the relationship between attention and visual short-term memory (VSTM). Specifically, we were interested in whether attention can influence representations already stored in VSTM. If attention affects representation maintained in VSTM, how does attention select these representations after the iconic image of stimuli has already faded? We examined two possible mechanisms underlying attentional selection from VSTM.

In the area of visual memory research, several studies have demonstrated that VSTM can hold or maintain a small number of items across the delay produced by an eye movement (Irwin, 1992; Irwin \& Andrews, 1996) or across a temporal delay without an eye movement (Luck \& Vogel, 1997; Vogel, Woodrow, \& Luck, 2001). In the latter case, a change-detection task is commonly used to assess the information stored in VSTM. In a typical changedetection task, observers are shown a memory array and a test array, separated by a brief delay interval, and observers are asked to report whether the two arrays were identical or different along one or more feature dimensions. When color is the relevant dimension, for example, the memory array contains a set of colored squares, and the test array is either identical to the memory array or differs in the color of one item. Accuracy is typically high when there are only one to three objects on the memory array and lower when the number of objects in the memory array increases, suggesting that VSTM has a capacity of approximately three or four objects.

Attention is widely believed to play an important role in VSTM (Bundesen, 1990; Cowan, 1997; Duncan \& Humphreys, 1989). Many studies have shown that attentiondirecting cues can influence which items are encoded in VSTM if the cues appear before stimulus offset, or before the iconic image of the stimulus has faded (Averbach \& Coriell, 1961; Becker, Pashler, \& Anstis, 2000; Griffin \& Nobre, 2003; Landman, Sperkreijse, \& Lamme, 2003; Schmidt, Vogel, Woodman, \& Luck, 2002; Sperling, 1960; Woodman, Vecera, \& Luck, 2003). In these cases, it is thought that attention selects a perceptual representation for entry into VSTM (see Schmidt et al., 2002; Vogel, Woodrow, \& Luck, 2006).

Although attention can influence the storage of items in VSTM when attention is directed to an object before it appears (or shortly after it disappears), many findings suggest that attention does not influence VSTM when attention is directed long after the presentation of objects (i.e., beyond the range of iconic memory; Averbach \& Coriell,

M. Matsukura, michi-matsukura@uiowa.edu 
1961; Becker et al., 2000; Sperling, 1960). In Sperling's partial report procedure, for example, three rows of alphanumeric characters were presented briefly, followed by a delay and a report tone that indicated which row of letters to report. The observers' task was to report the characters from the cued row. Sperling found that the iconic trace of the memory array had largely faded after $500 \mathrm{msec}$, and no cuing effect was observed when auditory cues were presented $1 \mathrm{sec}$ after the array offset. Averbach and Coriell replicated Sperling's finding with visual rather than auditory cues. Recently, Becker et al. found a significant cuing effect when their cue was presented at $215 \mathrm{msec}$ after the presentation of visual stimuli (letters, symbols, or colors) but not at $281 \mathrm{msec}$. Together, these results suggest that, if the object is to be remembered more accurately than uncued objects, attention must be directed to an object's location within $500 \mathrm{msec}$ after the object has disappeared.

All of these studies used stimuli that could be easily converted into a verbal code, and the observers were asked to verbally report the names of the stimuli. Thus, it was possible that attention controlled encoding of visual stimuli into verbal working memory rather than selecting items from VSTM. However, two recent studies of VSTM refuted a verbal recoding interpretation by demonstrating that cued objects are recalled more accurately than are uncued objects in change-detection tasks not requiring a verbal report. These change-detection tasks were coupled with a concurrent articulatory suppression task that interfered with visual-to-verbal recoding. Under such conditions, Schmidt et al. (2002) found a cuing effect with a cue that appeared immediately after the offset of the memory array. Similarly, Woodman et al. (2003) found a cuing effect with a cue that appeared $50 \mathrm{msec}$ after the offset of the memory array. These two studies demonstrate that attention can influence the encoding objects into VSTM if an attention-directing cue occurs before the perceptual representation of the memory array has faded.

In contrast to this view (that an attention cue plays a role before the iconic image of the memory array has faded), recent work by Griffin and Nobre (2003; see also Landman et al., 2003) found a significant cuing effect in a color change-detection task, even though cues were presented 1.5 to $2.5 \mathrm{sec}$ after the offset of the memory array, a time delay well beyond the range of iconic memory. In Experiment 2 from Griffin and Nobre (2003), observers were shown a memory array of four colored Xs and memory was tested after a delay of 2,000-3,500 msec by presenting a single item. The single probe item was presented for $100 \mathrm{msec}$ at the location of the corresponding memoryarray item. Observers made a speeded buttonpress response to indicate whether or not this test item was the same color as the corresponding item from the memory array. A central arrow was presented for $1,500-2,500 \mathrm{msec}$ after the memory array offset, during the retention interval. The cued location was tested for $80 \%$ of trials (valid trials), and an uncued location was tested on $10 \%$ of trials (invalid trials). A nonpredictive cue was presented on the remaining $10 \%$ of trials (neutral trials). Observers were fastest and most accurate on valid trials, somewhat slower and less accurate on neutral trials, and even slower and less accurate on invalid trials. This finding suggests that attention enhanced the representation of the validly cued item relative to the representation of the neutrally cued and invalidly cued items. Thus, Griffin and Nobre concluded that attention could influence and enhance the representations of objects stored in VSTM.

Although interesting, Griffin and Nobre's (2003) results are puzzling for two reasons. First, these findings conflict with previous studies that were discussed above, in which cues became ineffective a few hundred milliseconds after the memory array offset, presumably because there was no remaining perceptual representation of the object for the cues to encode into VSTM. Second, it is not clear how attention can enhance the representation of the cued item compared with neutral trials, again because there is no perceptual representation for attention to enhance. If there is no perceptual representation, such as an iconic image of input, how can attention enhance the representation of the validly cued item rather than the neutrally cued item? Given that no perceptual information was available for the cue to enhance, it seems plausible that the improved performance on valid trials could have been the result of some other kind of process. For example, the cue might have protected a memory representation from some kind of disruption, such as passive decay or interference from other items in VSTM. Alternatively, attention might have assisted the comparison process between items in memory and the test probe: Observers might have compared cued items to the test probe before comparing uncued items. Also, there are more mundane explanations why observers performed more accurately on valid trials than on neutral trials. Observers might have made eye movements to the cued locations, and these eye movements may have improved the perception of the test item at the cued location.

Before addressing the source of attentional selection from VSTM, it was necessary to rule out the latter eye movement explanation. In Griffin and Nobre's (2003) study, the test probe appeared for only $100 \mathrm{msec}$, making it possible that eye movements to the cued location improved the perception of the test item when the test item appeared at the cued location. Specifically, if observers were fixating the cued location at the time of the test-array presentation, they would have been able to perceive the color of the test item when it appeared at the cued location (i.e., on valid trials). Perception of the test item would have been poorer on neutral trials, assuming that the eyes remained at center on these trials and that the test item was therefore presented parafoveally. Perception of the test item would have been even poorer on invalid trials, presented at a distant uncued location. Because the test probe appeared for only $100 \mathrm{msec}$, observers would not have sufficient time to move their eyes to the test item on neutral and invalid trials.

To examine the role of eye movements on attention effects in memory, Experiments 1 and 2 used a paradigm similar to that of Griffin and Nobre (2003) but included a test-display duration of 2,000 msec. Presenting the test display for 2,000 msec would provide ample opportunity for observers to fixate the test item even on neutral and invalid trials. If eye movements were responsible for the ef- 
fects observed by Griffin and Nobre, these effects should be eliminated with the 2,000-msec test duration.

To anticipate our results, Experiments 1 and 2 ruled out the possibility that eye movements were responsible for the observed cuing effect. Consequently, subsequent experiments examined two mechanisms for how attention could benefit items stored in VSTM. One explanation for the attentional benefits observed by Griffin and Nobre (2003) is that attention protects the representation of the cued item from decay, interference, or some other kind of degradation that might occur during the retention interval. According to this account, representations in VSTM become progressively less accurate over the course of the retention interval, but attending to a single item can reduce the rate or probability of degradation. Under this account, attending to a VSTM representation makes the representation stronger, allowing it to survive the retention interval so that it can be accurately matched with the test item. We term this possibility the protection account because attention protects the cued item from degradation. A second possibility is that attention could act to prioritize the order of comparison of items in VSTM to items in the test display. To perform change detection, items in memory must be compared with items in the test probe, and this process may be prone to errors. Attention could assist this comparison process by biasing observers to first compare the cued object with the test display; only after this comparison failed would other items in memory be compared with the test display. Under this account, priority is given to cued items. On valid trials, the test item matches the priority setting, but on neutral or invalid trials the test item mismatches the priority setting. The priority setting must be altered on these trials, and this could produce a decline in accuracy. We term this possibility the prioritization account because attention selects the order of (i.e., prioritizes) comparison to items stored in memory (see Johnson \& Yantis, 1995, and Shomstein \& Yantis, 2002, 2004, for further discussion of the role of attention in setting priority levels). The protection and prioritization accounts were compared in Experiments 3, 4, and 5.

\section{EXPERIMENT 1}

In Experiment 1, we attempted to rule out the possibility that the observed attentional benefit for cued objects in VSTM was caused by eye movements toward the cued location, which resulted in improved perception of the test item. To examine this possibility, we compared $100-\mathrm{msec}$ and 2,000-msec test durations. If the cue validity effects observed by Griffin and Nobre (2003) were a result of covert shifts of attention, the duration of the test probe should not matter, but if the validity effects were a result of eye movements, such effects should disappear with a long-duration test probe. This is because observers could refixate even if they had initially fixated the cued location.

This experiment also used cuing procedures that were somewhat different from those of Griffin and Nobre (2003). Most notably, we used only valid and neutral cues because invalid cues do not readily distinguish the different effects of attention. For example, one could imagine that observers intentionally forget the uncued item due to the demand characteristics of the experiment, which makes the impaired performance on invalid trials somewhat difficult to interpret. We also made the valid and neutral trials equiprobable in the present experiments, which increased statistical power.

In Experiment 1, we tested two different set sizes, 4 and 6 , in separate groups of observers. The set size manipulation was included to ensure that our conclusion regarding attentional selection from VSTM would generalize across set sizes near and above the capacity of VSTM ( 3 or 4 items).

\section{Method}

Participants. For each set-size condition, 15 University of Iowa undergraduates (age range, 18 to 30) participated to receive partial course credits for their involvement; all reported having normal or corrected-to-normal visual acuity. None of the present observers had participated in any of the other experiments reported in the present article.

Stimuli. Stimuli were viewed from a distance of $60 \mathrm{~cm}$ and were presented on a gray background $\left(22.6 \mathrm{~cd} / \mathrm{m}^{2}\right)$ with a continuously visible white fixation cross $\left(51.5 \mathrm{~cd} / \mathrm{m}^{2}\right)$. For a set size of 6 , the stimuli were presented at six locations evenly spaced around an imaginary circle, with a radius of $3.8^{\circ}$, that was centered at fixation (see Figure 1). Each memory array consisted of a $1.1^{\circ} \times 1.1^{\circ}$ filled square at each of the six locations. The set-size-4 display was identical to the set-size- 6 display, except that four locations were evenly spaced around the imaginary circle. The squares' colors were selected at random (without replacement) from a set of seven easily discriminable colors: violet $\left(x=.245, y=.111,6.4 \mathrm{~cd} / \mathrm{m}^{2}\right), \operatorname{red}(x=$ $\left..636, y=.315,12.9 \mathrm{~cd} / \mathrm{m}^{2}\right)$, blue $\left(x=.152, y=.659,5.6 \mathrm{~cd} / \mathrm{m}^{2}\right)$, green $\left(x=.313, y=.554,20.2 \mathrm{~cd} / \mathrm{m}^{2}\right)$, yellow $(x=.464, y=.451$, $\left.38.14 \mathrm{~cd} / \mathrm{m}^{2}\right)$, black $\left(x=.299, y=.255, .5 \mathrm{~cd} / \mathrm{m}^{2}\right)$, and brown $\left(x=.582, y=.310,3.1 \mathrm{~cd} / \mathrm{m}^{2}\right)$. The central cue was a white arrow $\left(51.5 \mathrm{~cd} / \mathrm{m}^{2}\right), 1.9^{\circ}$ in length.

Procedure. Each trial began with an observer performing an articulatory suppression task, in which the observer was required to repeat either "A, B, C, D" or " $1,2,3,4$ " aloud through the duration of the trial. This concurrent task effectively discouraged verbal recoding of visual information (Baddeley, 1986; Besner, Davies, \& Daniels, 1981; Murray, 1968). Observers were instructed to speak at a rate of 3 or 4 digits/sec or 3 or 4 letters/sec, and the experimenter continuously monitored the observers to ensure adequate performance.

As depicted in Figure 1, the memory array appeared for a duration of $100 \mathrm{msec}$ after a 1,000-msec fixation screen. The offset of the memory array was followed by a blank delay period randomly selected out of 1,500,2,000, and 2,500 msec; then a cue appeared for $100 \mathrm{msec}$. The offset of the cue was followed by another blank period that varied randomly within the range from 500 to $1,000 \mathrm{msec}$, then the test probe was presented for either 100 or $2,000 \mathrm{msec}$. On valid trials, the cue pointed to one of the four or six locations that had been occupied by a color square in the memory array, and the test item always appeared at this location. On neutral trials, the cue was a set of four or six arrows that pointed to each of these four or six locations, and the test item could appear at any of these locations with equal probability. Valid and neutral cues were each $50 \%$ probable and varied unpredictably from trial to trial. On half of the trials, the probe square was the same color as the memory array square; on the remaining trials, the probe square was selected at random from the colors that had not been present in the memory array. The observers made an unspeeded manual response on a Cedrus 4 button response box to indicate whether the color of the test item matched the color of the corresponding item from the memory array.

Each observer performed a single experimental session. At the beginning of the session, the observers were given both written and verbal instructions. After a few minutes of practice with the task, 


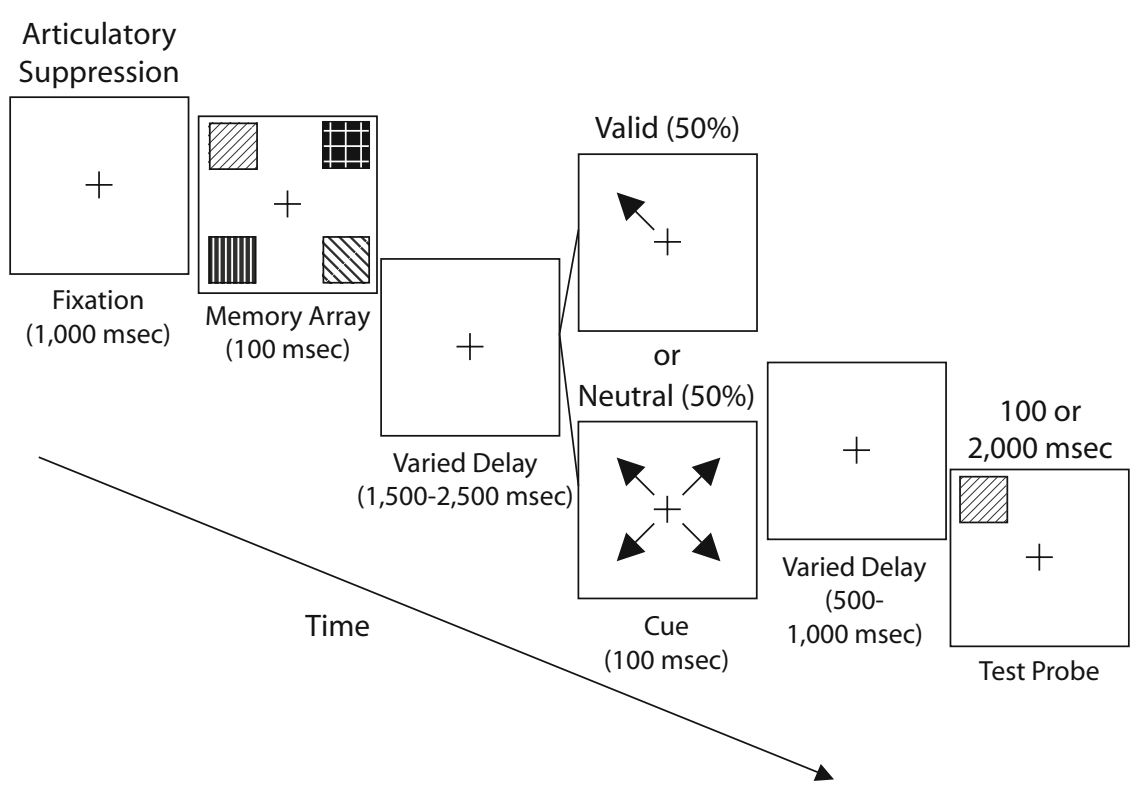

Figure 1. Example of the procedure used in Experiment 1 (set size 4). For illustrative purposes, the stimuli are drawn much larger than they appeared in the actual computer display; also, different fill patterns are used to represent different colors and the fixation and arrow cues were presented in white on a gray background.

each observer completed six blocks of 84 trials. Test item duration was $100 \mathrm{msec}$ on three blocks and 2,000 msec on three blocks. These two types of blocks were alternated, and the starting duration was counterbalanced across observers.

\section{Results and Discussion}

Figure 2 shows accuracy (percent correct, collapsed across change and no-change trials) for valid and neutral trials for Experiment $1 .{ }^{1}$ For both set sizes and both testprobe durations, accuracy was greater on valid trials than on neutral trials. This cue validity effect was somewhat larger for the 2,000-msec duration than for the $100-\mathrm{msec}$ duration, and it was also somewhat larger for set size 4 than for set size 6 .

These observations were supported by an ANOVA with within-subjects factors of cue type (valid vs. neutral) and test-array duration (100 vs. $2,000 \mathrm{msec}$ ), and a betweensubjects factor of set size (4 vs. 6). Greater accuracy on valid trials than on invalid trials led to a significant main effect of cue type $[F(1,28)=135, p<.0001]$, and greater accuracy at set size 4 than at set size 6 led to a significant main effect of set size $[F(1,28)=6, p<.02]$. Accuracy did not significantly differ between 100 -msec and 2,000-msec test-array durations $[F(1,28)=2, p>.2]$. The larger cuing effect at set size 4 led to a significant cue type by set size interaction $[F(1,28)=8, p<.008]$, and the larger cuing effect for the $2,000 \mathrm{msec}$ duration led to a significant cue type by duration interaction $[F(1,28)=10, p<.003]$. The three-way interaction of cue type, test-array duration, and set size was not significant $[F(1,28)=2, p>.2]$.

The data from the two set sizes were also analyzed separately. The cue-type main effect was significant for both
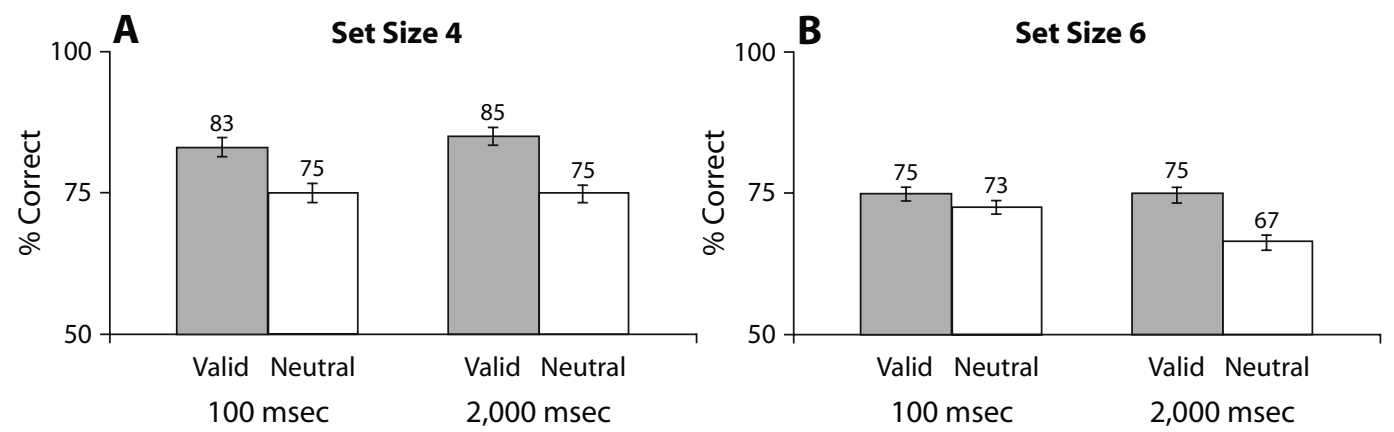

Figure 2. Change-detection accuracy from Experiment 1. (A) Data from set size 4. (B) Data from set size 6. The $x$-axis represents cue type (valid vs. neutral) and test-probe duration (100 vs. $2,000 \mathrm{msec}$ ), whereas the $y$-axis stands for percentage of correct trials. For this and all subsequent figures, error bars represent $95 \%$ withinsubjects confidence intervals (Loftus \& Masson, 1994). 
set-size- 4 and set-size- 6 conditions ( $p<.0001$ for both). Planned pairwise comparisons indicated that this effect was significant for both the 100 and 2,000-msec test-array durations at both set sizes ( $p<.03$ for the 100 -msec duration at set size $6 ; p<.0001$ for the other cases). The cuevalidity effect was clearly larger for the 2,000 -msec duration than for the 100 -msec duration at set size 6 , leading to a significant cue type by duration interaction $(p<.005)$; but this interaction was smaller and did not approach significance at set size 4 . However, given the absence of a three-way interaction between set size, cue type, and duration in the omnibus ANOVA, we cannot draw any conclusions about this difference between the two set sizes.

The results of Experiment 1 indicate that the cuevalidity effects observed by Griffin and Nobre (2003) were, in fact, a result of covert shift of attention. Specifically, robust cuing effects were observed at the 2,000-msec test-array duration for both set sizes, even though shifts of gaze could be made on both valid and neutral trials. The results rule out eye movements during the retention interval as an explanation for the prior results and indicate that covert attention can influence representations of items already stored in VSTM.

\section{EXPERIMENT 2}

Although the results of Experiment 1 ruled out an eye movement account of cuing effect in VSTM, an alternative remains: Eye movements could have been executed at the long delays in Experiment 1 and might have interfered with the comparison between items in memory and the test probe. In short, corrective eye movements would be more likely to occur on neutral trials than on valid trials, and these eye movements could have disrupted performance on neutral trials.

In Experiment 2, we attempted to rule out this alternative eye movement account by monitoring observers' eye movements. This experiment used trials with a 2,000-msec test duration and a set size of four items. If large cuing benefits are observed in the absence of eye movements, this will demonstrate that the covert attention can influence the representations of items stored in VSTM.

\footnotetext{
Method

Participants. Six University of Iowa students volunteered to participate. All reported having normal or corrected-to-normal visual acuity.

Stimuli and Procedure. The stimuli and procedure were identical to those of Experiment 1, except that (1) the duration of the test probe was always 2,000 msec; (2) the set size was four items; (3) observers initiated each trial by pressing a key with the right hand; and (4) observers received a total of 124 trials with 12 practice trials at the beginning of the session.

Eye position was measured with an Applied Science Laboratories Eye Trac Model 210. All observers were instructed to maintain fixation because trials with eye movements would be removed from the data analysis. Eye movements were monitored by the experimenter, who kept a trial-by-trial record of whether an eye movement had occurred. Eye movements were monitored from the first keypress that started the trial to the keypress that observers made in responding to the target. Eye movements were defined as a movement of more than $1^{\circ}$ to the left or right of fixation.
}

\section{Results and Discussion}

Mean accuracy of each observer was calculated, as in Experiment 1. Fewer than 1\% of the trials were removed due to eye movements. Accuracy was significantly greater on valid trials (91\%) than on neutral trials $(84 \%)[t(5)=7$, $p<.005]$. This $7 \%$ effect was nearly identical in size to the $8 \%$ effect observed on the comparable trials on Experiment $1 .^{2}$ Together with the results of Experiment 1, these results provide strong evidence that shifts of covert attention - in the absence of overt gaze shift - can influence representations of items stored in VSTM as proposed by Griffin and Nobre (2003).

\section{EXPERIMENT 3}

Having verified that attention can influence representations of objects stored in VSTM, we now turn to the more theoretically important issue regarding possible mechanisms of the attentional selection. As described in the introduction, we contrasted a protection account and a prioritization account. According to the protection account, attention could protect the cued item from decay, interference, or some other kind of degradation that might occur during the retention interval. According to the prioritization account, attention could give the cued item higher priority during the process of comparing the VSTM representation of the memory array to the test item.

To distinguish these two alternatives, we developed a double-cue procedure, in which observers were first cued to one side of the display, then occasionally cued to the other side (see also Landman et al., 2003, for a similar procedure). When the second cue appeared, it was $100 \%$ predictive of the items that would be tested. The logic of this procedure, depicted in Figure 3, is as follows: If observers were cued to one side, the protection hypothesis would predict that the information from the uncued side would become degraded either by interference from other items in memory or by decay. Directing the observer's attention to the other side at that point would not be useful because memory for these items had already become degraded. Thus, observers should be more accurate when they were tested for the items cued first than for the set of items cued second. By contrast, the prioritization hypothesis predicts that the cue would change the priority given to the cued items during the comparison process. Because the second cue was perfectly predictive of the set of items that would be tested, a prioritization account predicts that observers should give processing priority to the items cued second; therefore, a prioritization account predicts that observers would be equally accurate when tested for second-cued and first-cued items.

\section{Method}

Participants and Stimuli. As in Experiment 1, we tested set size 4 and set size 6 in separate groups of observers. The method for Experiment 3 was the same as that for Experiment 1, with the following exceptions: Twenty new participants served as observers for each set size condition. For a set size of 6,3 stimuli on a given side in the memory array were positioned in a vertical line $3.8^{\circ}$ lateral to the vertical meridian and spaced $2.3^{\circ}$ vertically. For a set size of 4 , two stimuli on a given side were presented in the memory array. 

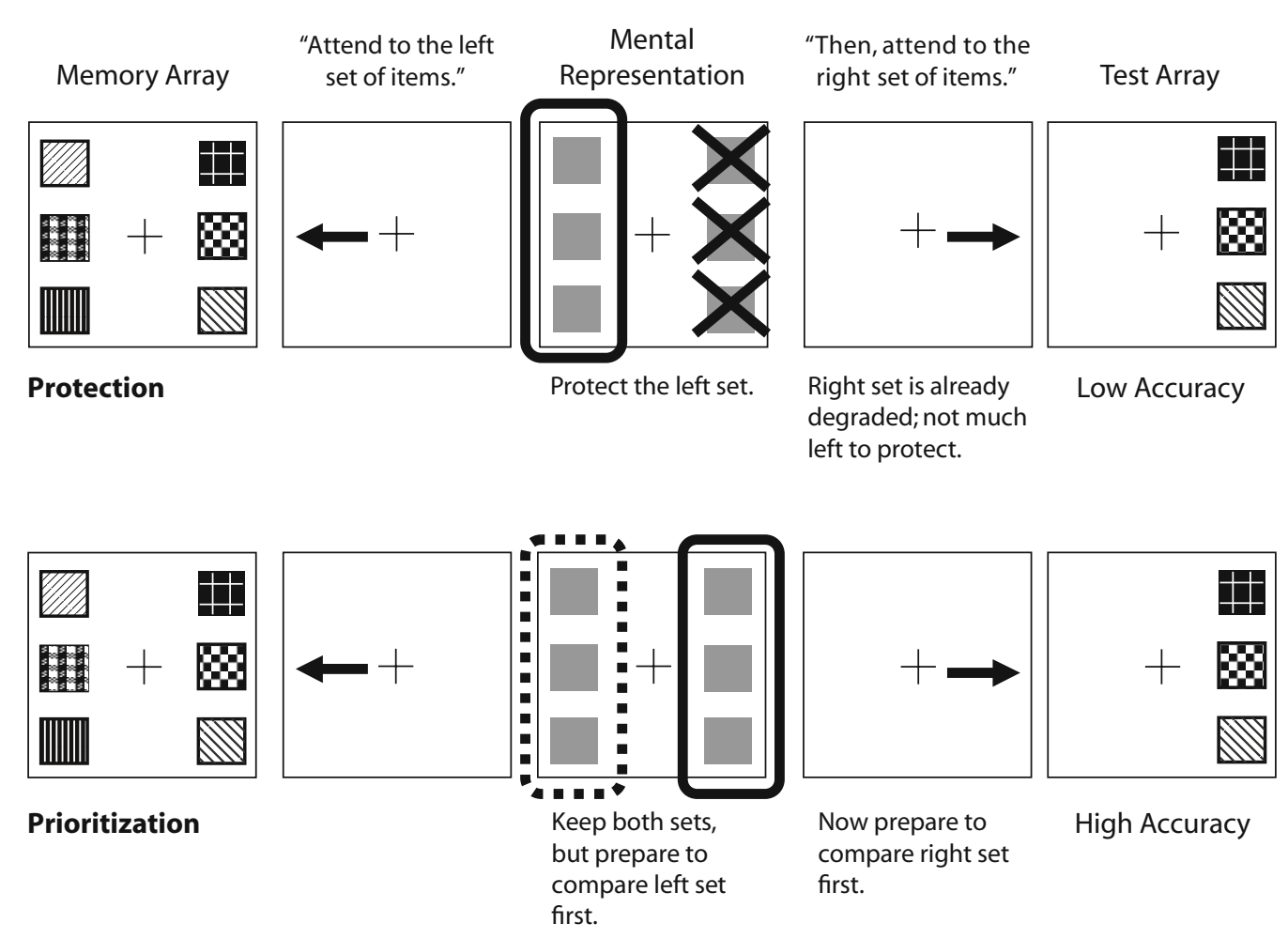

Figure 3. Illustration of the two attentional mechanisms in VSTM.

The cue was an arrow that pointed either directly leftward or directly rightward from the fixation point, cuing an entire hemifield. The test array contained either the left subset of color squares or the right subset.

Design. As illustrated in Figure 3, the memory array contained two sets of either two or three colored squares, one on each side of the display, and the test array consisted of either two or three items on a single side. This test array was either identical to the three items that had been presented on that side in the test array $(50 \%)$ or differed in the color of one item (50\%). On two thirds of trials, an arrow during the retention interval cued observers to attend to one side (single-cue trials). Within these single-cue trials, the test array was presented on the cued side on valid trials $(80 \%)$, and the test array was presented on the uncued side on invalid trials $(20 \%)$. Both the protection and prioritization hypotheses would predict higher accuracy on valid trials than on invalid trials from the single-cue condition.

The remaining third of trials were cued by a sequence of two arrows during the retention interval (double-cue trials). On two thirds of these double-cue trials, both cues pointed to the same side (samedirection trials). On the remaining third of the double-cue trials, the second cue pointed to the opposite side from the first cue (differentdirection trials). On double-cue trials, the second cue indicated the position of the subsequent test item with $100 \%$ validity. The observers were informed of this and therefore were highly motivated to attend to the side indicated by this second cue. However, they could not know if a trial was going to be single-cue or double-cue until a second cue was presented; consequently, they had to attend to the side indicated by the first cue until a second cue was presented. Our design included single-cue trials to prevent observers from ignoring the first cue and selecting items based on the second cue alone. The optimal strategy would therefore be to attend to the side indicated by the first cue, then to switch to the other side if a second cue pointed to the opposite direction (which occurred on a ninth of all trials). This strategy would be effective only if attention operated by means of prioritization, not of protection.
The reasoning for this prediction is illustrated in Figure 3, which shows a double-cue trial with the first cue pointing to the left and the second cue pointing to the right (i.e., the different-direction trial). According to the protection account (depicted on the top in Figure 3), attention will protect from decay and interference the VSTM representation of the items from the left side. Consequently, the VSTM representation of the items from the right side should become degraded. When the second cue appears, signaling the observer to shift attention to the VSTM representation of the items on the right side, the representation on the right side will already be degraded. Thus, there is nothing attention can do to improve these already degraded representations; that is, there is no source of information about the colors of these items other than the already degraded VSTM representations, so attention cannot improve the representation to the same level that would be obtained when both cues point in the same direction (i.e., the same-direction trial). Note that Experiment 5 will demonstrate that observers are fully capable of switching attention for double-cue trials under more typical cuing conditions.

By contrast, the prioritization account (depicted at the bottom of Figure 3) predicts that observers should be able to shift attention to the other side during the retention interval. When the first cue points to the left, the left-side items are given higher priority for comparison, but the representations of the left-side and right-side items are retained equally well in VSTM. When the second cue points to the opposite direction, it is therefore possible to switch prioritization. Thus, accuracy should be approximately equal for same-direction and different-direction double-cue trials.

Procedure. Single-cue trials (67\% of trials) and double-cue trials ( $33 \%$ of trials) were randomly intermixed. The event sequence for a single-cue trial is depicted in Figure 4A. After a 1,000-msec fixation screen, the memory array appeared for $150 \mathrm{msec}$. The offset of the memory array was followed by a blank period of 1,450 msec; then the cue was presented for $150 \mathrm{msec}$. The test array appeared on the cued side on $80 \%$ of the single-cue trials and on the uncued side on $20 \%$ of the single-cue trials. The offset of the single cue was followed 


\section{A}

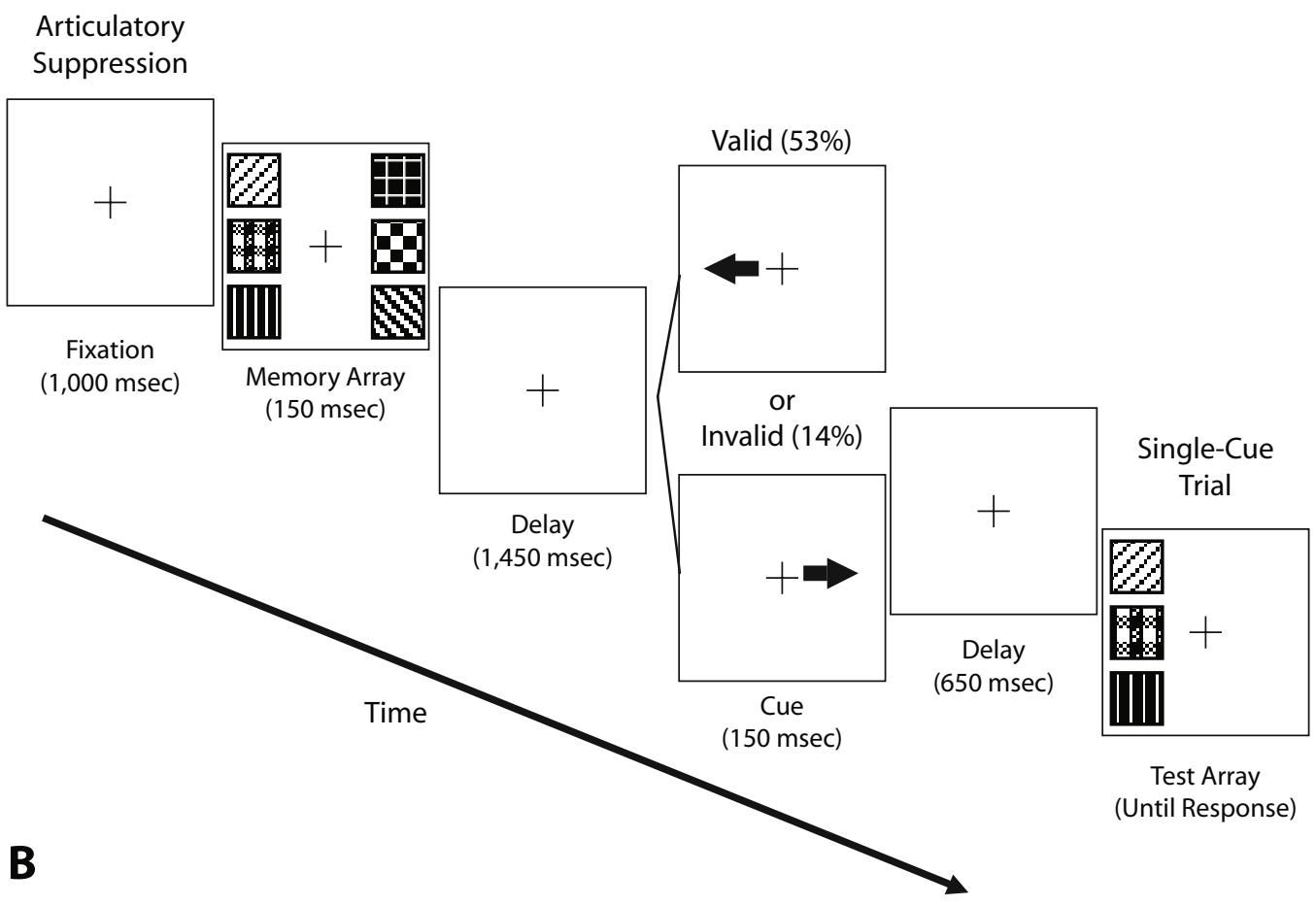

Articulatory Suppression

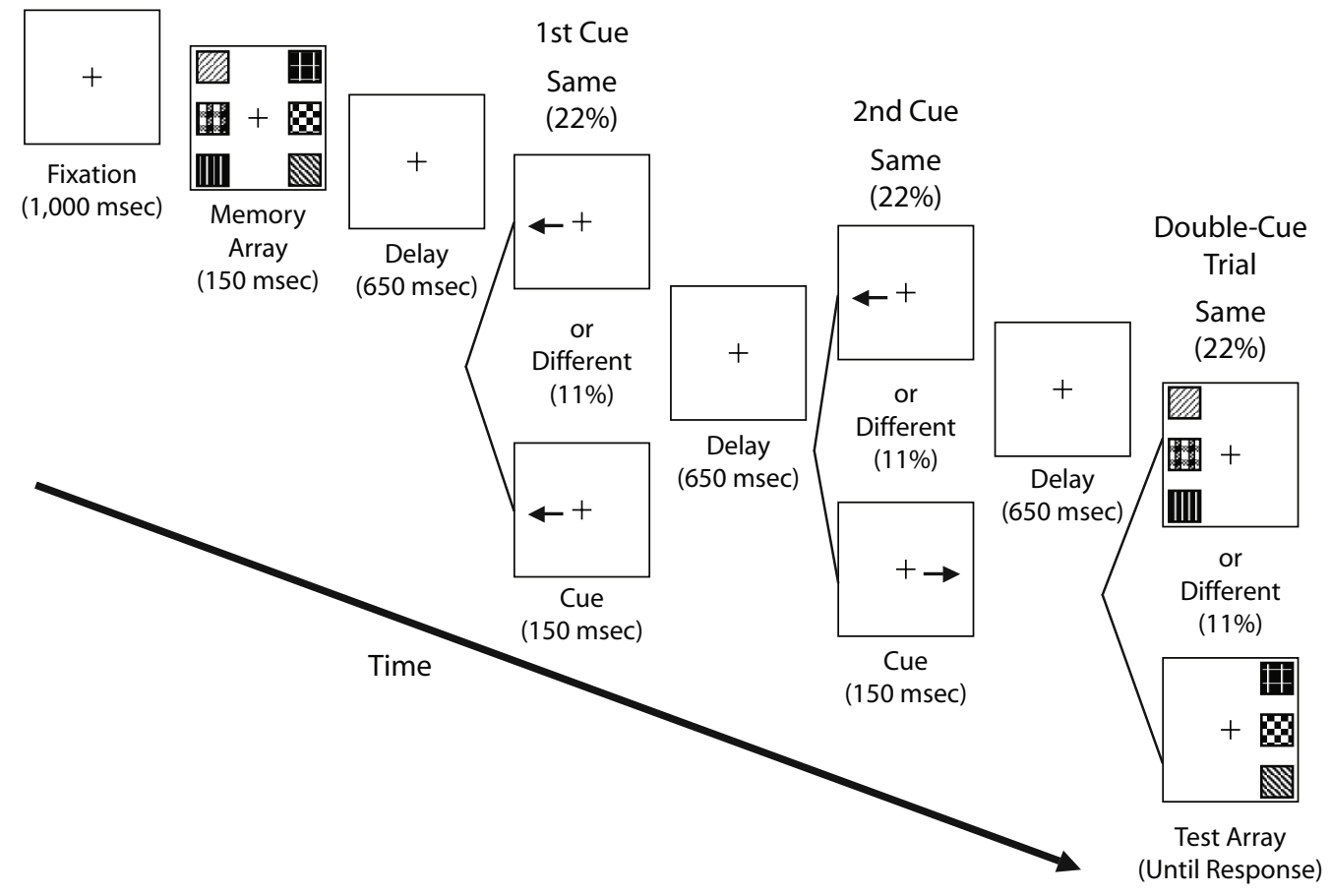

Figure 4. (A) Event sequence for a single-cue trial in Experiment 3. (B) Event sequence for a double-cue trial in Experiment 3.

by another blank period of $650 \mathrm{msec}$ and the test array. The test array was presented and remained visible until the observer responded.

The event sequence for a double-cue trial is depicted in Figure 4B. These trials were identical to single-cue trials, except that an addi- tional cue was presented and the second cue was $100 \%$ valid. The second cue of double-cue trials appeared at the timing that corresponded to the timing of the sole cue of single-cue trials. The first cue appeared for $650 \mathrm{msec}$ after the offset of the memory array and 

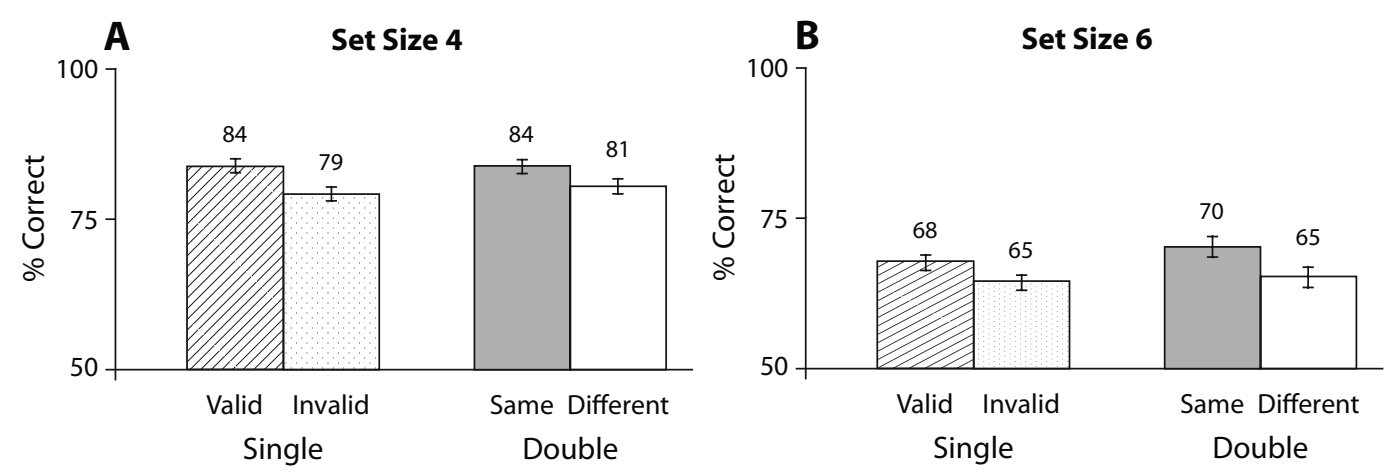

Figure 5. Change-detection accuracy from Experiment 3. (A) Data from set size 4. (B) Data from set size 6. The $x$-axis represents cue type, single-cue types on the left and double-cue types on the right. The $y$-axis stands for percentage of correct trials.

was presented for a duration of $150 \mathrm{msec}$. The second cue was presented for $650 \mathrm{msec}$ after the offset of the first cue. The total retention interval was the same on single-cue and double-cue trials. The second cue pointed in the same direction as the first cue with $67 \%$ of double-cue trials and pointed the opposite side with $33 \%$ of doublecue trials. The observers were informed of all probabilities. All four types of trials were mixed within six blocks of 84 trials.

\section{Results and Discussion}

Figure 5 shows accuracy (percent correct) for singlecue and double-cue trials at set sizes 4 and 6 . Because the single-cue condition was subdivided into valid and invalid trials and the double-cue condition was subdivided into two kinds of valid trials - same-direction and differentdirection trials - we conducted separate ANOVAs for single-cue and double-cue trials. The ANOVAs contained a within-subjects factor of cue type (valid vs. invalid for the single-cue condition; same-direction vs. different-direction for the double-cue condition) and a between-subjects factor of set size (4 vs. 6). For both set sizes, accuracies were greater on valid trials than on invalid trials for the singlecue condition and greater on same-direction trials than on different-direction trials for the double-cue condition.

For the single-cue trials, greater accuracy on valid trials than on invalid trials led to a significant main effect of cue type $[F(1,38)=22, p<.0001]$, and greater accuracy at set size 4 than at set size 6 led to a significant main effect of set size $[F(1,38)=83, p<.0001]$. The single-cue validity effect indicates that observers used the first cue to direct attention to one of the two sets of objects. The magnitude of this single-cue validity effect did not significantly differ between two set sizes $[F(1,38)=0.7, p>.4]$.

For the double-cue trials, greater accuracy on samedirection trials than on different-direction trials led to a significant main effect of cue type $[F(1,38)=17, p<$ $.0001]$. Greater accuracy at set size 4 than set size 6 led to a significant main effect of set size $[F(1,38)=62$, $p<.0001]$. The interaction between double-cue type and set size did not approach significance $[F(1,38)=0.7$, $p>.4]$. These results suggest that observers shifted attention, as indicated by the first cue, but were unable to use the second cue to redirect attention. The current pattern indicates that attending to one side leads to a loss of information from the other side. Thus, observers could not regain the information when the second cue indicated that they should shift their attention to the other side. Because the cue type by set size interaction was not significant for either the single-cue condition or the double-cue condition, we cannot draw any conclusions about the difference between the two set sizes.

These results support the hypothesis that attention serves to protect the attended items from degradation or decay during the retention interval. When a second cue pointing in a different direction from the first cue appeared, the items that were first uncued became degraded or decayed over time. When the second cue appeared, attention could not do anything to improve these degraded representations. The results of Experiment 2 are inconsistent with the hypothesis that attention simply changes the priority of the items in the comparison process. It should be noted that performance on same-direction double-cue trials was as good as, or better than, performance on single-cue trials. This indicates that the presence of two cues did not lead to any general disruption of performance.

\section{EXPERIMENT 4}

Although the results of Experiment 3 were consistent with the protection pattern, an alternative explanation must be ruled out. Specifically, the cue on single-cue trials was presented $1,450 \mathrm{msec}$ after the offset of the memory array, whereas the first cue on double-cue trials appeared at $650 \mathrm{msec}$ after the offset of the memory array. If the observers noticed this regularity, they might have been able to determine whether a trial was a single-cue or double-cue trial when a cue was presented at $650 \mathrm{msec}$. This may have led to differences in strategy on single-cue and double-cue trials.

A plausible strategy would be simply to ignore the spatial information provided by the first cue on single-cue trials (because the second cue was $100 \%$ valid). However, this would have led to no difference in performance between same-direction double-cue trials and different-direction double-cue trials (as predicted by the prioritization account). However, we observed a significant difference be- 
tween these two types of double-cue trials. Nevertheless, because it is not possible to anticipate all possible strategies, Experiment 4 was conducted to eliminate any possible strategic differences. In this experiment, single cues could appear either $650 \mathrm{msec}$ or 1,450 msec following the offset of the memory array, just like the cues in the doublecue condition. This procedure should encourage observers to direct their attention based on every cue that appeared during the retention interval. We predict that the results would be identical to those observed in Experiment 3.

\section{Method}

The method for Experiment 4 was the same as that for Experiment 3, with the following exceptions: (1) Twenty new participants served as observers; (2) only the set-size- 6 condition was used; (3) there were two types of single cues. Half of the single cues $(33 \%$ of the entire set of trials) were presented $650 \mathrm{msec}$ after the offset of the memory array, whereas the other half of the single cues $(33 \%$ of the entire set of trials) were presented $1,450 \mathrm{msec}$ after the offset of the memory array. The remaining $33 \%$ of trials were double-cue trials with cues at 650 and $1,450 \mathrm{msec}$ after the offset of the memory array.

\section{Results and Discussion}

Figure 6 shows accuracy (percent correct) for singlecue and double-cue trials. For single-cue trials, accuracy was greater on valid trials than on invalid trials regardless of whether the cue was presented with a delay of $650 \mathrm{msec}$ or 1,450 msec. An ANOVA for the single-cue trials with factors of delay period and cue validity showed a significant main effect of cue validity $[F(1,19)=23, p<$ $.0001]$, but no significant interaction between cue validity and delay period $[F(1,19)=0.3, p>.6]$. Follow-up analyses showed that the cuing effect for single-cue trials was significant for both the 650 -msec delay $[F(1,19)=5$, $p<.004]$ and the 1,450 -msec delay $[F(1,19)=23, p<$ $.0001]$. Thus, the observers shifted attention when a cue was presented at $650 \mathrm{msec}$, at which point they could not know whether the trial was single-cue or double-cue.

For the double-cue trials, greater accuracy was observed on same-direction trials than on different-direction trials, just as in Experiment 3. A one-way ANOVA with a factor of same-direction versus different-direction cues yielded

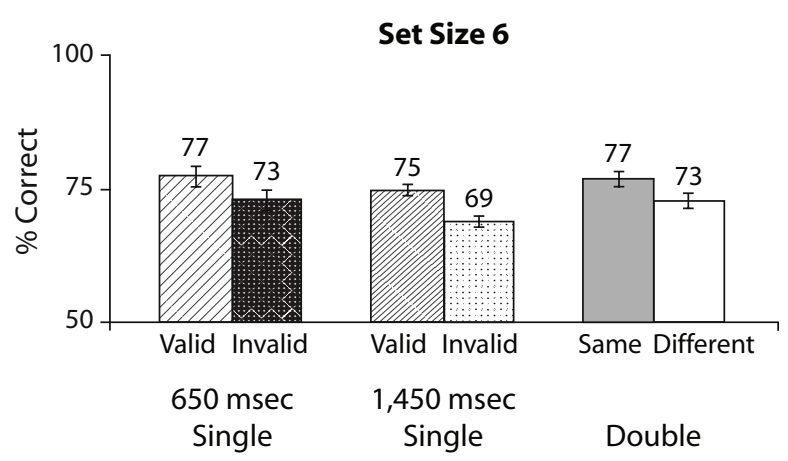

Figure 6. Change-detection accuracy from Experiment 4. The $x$-axis represents cue type, the 650-msec-delayed single-cue types on the left, 1,450-msec-delayed single-cue types on the center, and double-cue types on the right. a significant main effect $[F(1,19)=9, p<.0007]$. The overall pattern of results indicates that observers shifted attention to the location indicated by the first cue but were unable to use the second cue to redirect attention. Attending to one set of items apparently led to a loss of information from the other (uncued) set of items. These results provide further support for the proposal that attention can serve to protect cued representations from degradation.

\section{EXPERIMENT 5}

Although Experiments 3 and 4 suggest that cued items are protected in memory, observers may have been unwilling or unable to shift attention to one cued location and then to another; that is, the double-cuing procedure may have been too complicated or too rapid, leading observers to simply focus attention on the basis of the first cue, ignoring the second cue. It is therefore important to demonstrate that our observers can, in principle, execute a shift of attention in response to one cue and then an oppositedirection shift of attention in response to a second cue. Experiment 5 therefore examined double-cuing trials in the context of a more typical cuing paradigm (see Figure 7), in which the cue indicated the likely location of a target that required a simple speeded detection response (Posner, 1980). If observers could switch attention effectively on different-direction trials in this context, they should have been able to do so in Experiments 3 and 4. Experiment 5 will demonstrate that they have this ability.

It should be noted that observers in Experiments 3 and 4 were asked to switch attention rapidly while VSTM was full; this might have made attention switching more difficult. Experiment 5 therefore required them to switch attention while maintaining a concurrent VSTM load. Consequently, VSTM was full while they were switching their attention to the opposite direction from the first cue, just as in Experiments 3 and 4.

It should be also noted that we are not assuming that the same mechanisms of attention are involved in the VSTM task of Experiments 3 and 4 and the speeded-detection task of Experiment 5. Indeed, different selection mechanisms are probably used for these tasks (see review by Luck \& Vecera, 2000). However, a common set of attentional control mechanisms appears to be used across many different types of selection tasks (Wojciulik \& Kanwisher, 1999). Thus, Experiment 5 can determine whether observers are willing and able to use these control mechanisms to program up sequences of shifts of attention in response to the types of cues used in Experiments 3 and 4. Moreover, this experiment will determine whether these shifts are possible when VSTM is full.

Our predictions are as follows: On single-cue trials, observers should shift attention to the cued side, leading to shorter response times (RTs) when the probe is presented on the cued side than when the probe is presented on the uncued side. On same-direction double-cue trials, observers should shift attention to the side indicated by the first cue and maintain attention on that side following the second cue. As a result, RTs on same-direction doublecue trials (which are always valid) should be as short as 


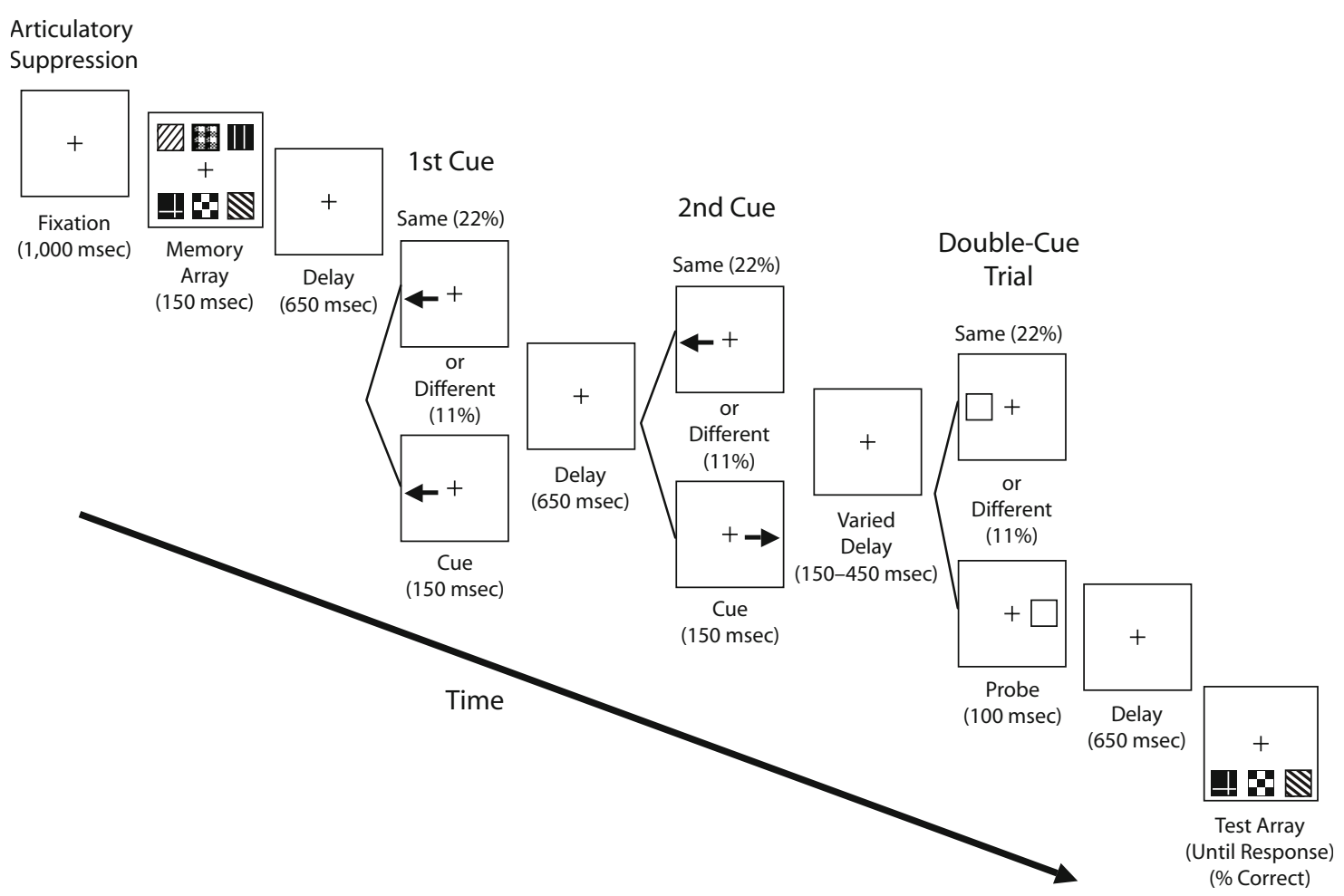

Figure 7. Event sequence for a double-cue trial of the probe-detection task used in Experiment 5.

those on single-cue valid trials. On the different-direction double-cue trials, observers should shift attention to the side indicated by the first cue and then switch attention to the opposite side when the second cue is presented. Again, because the probe is always presented on the side indicated by the second cue, RTs on these trials should be as short as those on same-direction double-cue trials and those on single-cue valid trials. This pattern would indicate that observers have the ability to switch attention under double-cue conditions.

In contrast, if observers cannot shift attention twice, they should shift attention to the side indicated by the first cue but be unable to switch attention to the opposite side on different-direction double-cue trials. Consequently, RTs on these different-direction trials would be longer than those on double-cue same-direction trials and on single-cue valid trials. This pattern would indicate that observers do not have the ability to switch attention under the double-cue condition.

\section{Method}

Participants. The observers were 10 University of Iowa undergraduates, who each received $\$ 8 / \mathrm{h}$ (age range, 18 to 30 years), and reported having normal or corrected-to-normal vision. None had participated in any of other experiments reported in the present article.

Stimuli. The background color, stimuli colors, and sizes were identical with those in the previous two experiments except for the spatial arrangement. The viewing distance changed from $60 \mathrm{~cm}$ to $100 \mathrm{~cm}$; however, the visual degree of each stimulus was adjusted to be identical with previous two experiments. In Experiment 5 , change detection was used as the cognitive load task. As illustrated in Figure 7, three squares appeared on the top of the fixation whereas three other squares appeared on the bottom. The test array contained only one set of the items, either on the top or the bottom of the fixation. The white box for the probe-detection task was $1.2^{\circ}$ subtending to each side. The probe was presented $3.8^{\circ}$ from the fixation point.

Procedure. The probability of four types of cued trials was identical with Experiment 3;66\% of the whole trials were precued by a single arrow, and $33 \%$ by double arrows. Within these single-cue trials, $80 \%$ were valid and $20 \%$ were invalid. Note that these percentages were for the main probe-detection task, not for the changedetection task. In Experiment 5, change detection was a secondary task to occupy observers' VSTM while observers were engaged in the main probe-detection task, in which they were required to press the button on the side on which a probe (the white box) appeared, as soon as they detected it.

For a single-cue trial, after the fixation screen for $1,000 \mathrm{msec}$ the memory array was presented for $150 \mathrm{msec}$ and followed by the blank period for $1,450 \mathrm{msec}$. The offset of this blank period was followed by the single cue, which predicted the probed side for the duration of $150 \mathrm{msec}$. The offset of the single cue was followed by another blank period, which randomly varied within the range from 150 to $450 \mathrm{msec}$; the white probe box appeared for $100 \mathrm{msec}$ and observers pressed the response button corresponding to the side on which the probe box appeared. As soon as observers made their detection response, another blank screen was presented for $650 \mathrm{msec}$ and the test array was presented until observers made their samedifferent response; as soon as they pressed the response button for the test array, the next trial started.

As illustrated in Figure 7, for a double-cue trial, after the fixation screen for $1,000 \mathrm{msec}$, the memory array appeared for $150 \mathrm{msec}$ and was followed by the blank period for $650 \mathrm{msec}$. At the offset of this blank period, the first precue was presented for $150 \mathrm{msec}$, followed by another blank period for the duration of $650 \mathrm{msec}$; then the second precue appeared for $150 \mathrm{msec}$. The offset of the second precue was followed by a blank period that randomly varied between 150 and $450 \mathrm{msec}$; the white box probe appeared for $100 \mathrm{msec}$, and 
observers reported its presence by pressing one of the response buttons. As soon as observers made their detection response, a blank screen appeared for $650 \mathrm{msec}$, and the test array was presented until observers made their same-different response.

As in Experiment 3, the duration between the memory-array offset and the precue onset was equated between single-cue and double-cue trials. Observers performed a total of 608 trials with short breaks for every 25 trials and a long break at the 300th trial. Each observer received a block of practice of 32 trials before the experimental session.

Aside from these loaded experimental trials, each observer performed separate change-detection only trials for a block of 32 trials, either before or after the main loaded experimental block as a control of memory performance. The trials were identical with those described above, except that observers performed the changedetection task only while ignoring the probe-detection task screens. Half of the observers went through the control block before the experimental trials started, and the other half did so after completing the experimental trials.

Eye movements were monitored with electrooculogram (EOG) recordings to ensure that observers did not simply fixate the cued location. The horizontal EOG was recorded as the voltage between electrodes placed from $1 \mathrm{~cm}$ lateral to the external canthi. The vertical eye EOG was recorded from an electrode beneath the left eye, and both electrodes were referenced to the average of reference electrodes located on the left and right mastoids. The EOGs were amplified by an SA Instrumentation amplifier with a gain of 20,000 and a bandpass of $0.01-80 \mathrm{~Hz}$, and the amplified signals were digitized at $250 \mathrm{~Hz}$ and averaged offline.

Blinks and eye movements (artifacts) were rejected through our standard two-step procedure (see Woodman \& Luck, 2003). In the first step, we calculated average horizontal individual trials containing artifacts. These trials were eliminated by calculating the cross covariance between the single-trial EOG waveform and a 100-msec step function; trials on which the maximum covariance exceeded a threshold (set separately for each observer) were rejected. In the second step, we calculated average horizontal EOG waveforms for left-cue and right-cue trials to examine the degree of residual eye movement activity. Any systematic eye movement toward the cued location can be observed in these waveforms with an extremely high ratio of signal to noise. Our analyses included only the data of observers whose residual EOG activity was less than $3 \mu \mathrm{V}$, which corresponds to an average eye movement of less than $0.2^{\circ}$. Incorrect trials and artifacts led to the rejection of an average of $36 \%$ of trials. Response speed was quantified as median RT for each observer to reduce the influence of outliers. Trials were collapsed across probe positions for all analyses.

\section{Results and Discussion}

The means of the median RTs are shown for each condition on the left side of Figure 8. As in Experiment 3, we performed separate ANOVAs for the single-cue and double-cue conditions. Overall accuracy for the probedetection was $99 \%$. In the single-cue condition, the mean of median RTs was $73 \mathrm{msec}$ faster on valid trials than on invalid trials $[F(1,9)=25, p<.001]$. In the doublecue condition, RTs for the same-direction and differentdirection cues were nearly identical and were quite similar to valid-trial RTs from the single-cue condition. The small difference between same-direction and different-direction trials in the double-cue condition was not statistically significant $[F(1,9)=0.005, p>.9]$.

The lack of a difference between same-direction and different-direction cues in the double-cue condition could indicate that the observers were simply ignoring the cues rather than shifting attention to the location indicated by the first cue and then shifting, if necessary, to the location indicated by the second cue. If that were true, the RTs on different-direction double-cue trials should be slower than the RTs from valid trials in the single-cue condition and similar to the RTs from invalid trials in the single-cue condition. If, in contrast, the observers shifted attention in response to the first cue and then shifted again on different-direction double-cue trials, then the RTs on these trials should be similar to those observed on valid trials in the single-cue condition. In fact, the RTs for the different-direction trials in the double-cue condition were just as fast as, or faster than, the valid trials in the single-cue condition. To provide statistical support for this observation, we compared the differentdirection double-cue trials with valid and invalid trials from the single-cue condition in separate one-way ANOVAs. RTs were significantly shorter on different-direction double-cue trials than invalid single-cue trials $[F(1,9)=53, p<.0001]$, and there was no significant difference between differentdirection trials from the double-cue condition and valid trials from the single-cue condition $[F(1,9)=2, p>.2]$. This pattern of results provides positive evidence that observers shifted attention to the location indicated by the second cue on double-cue trials. Moreover, because observers could not determine whether a trial would have one or two cues (as demonstrated in Experiment 4), the finding of a significant validity effect in the single-cue condition indicates that they also shifted attention to the location indicated by the first cue.
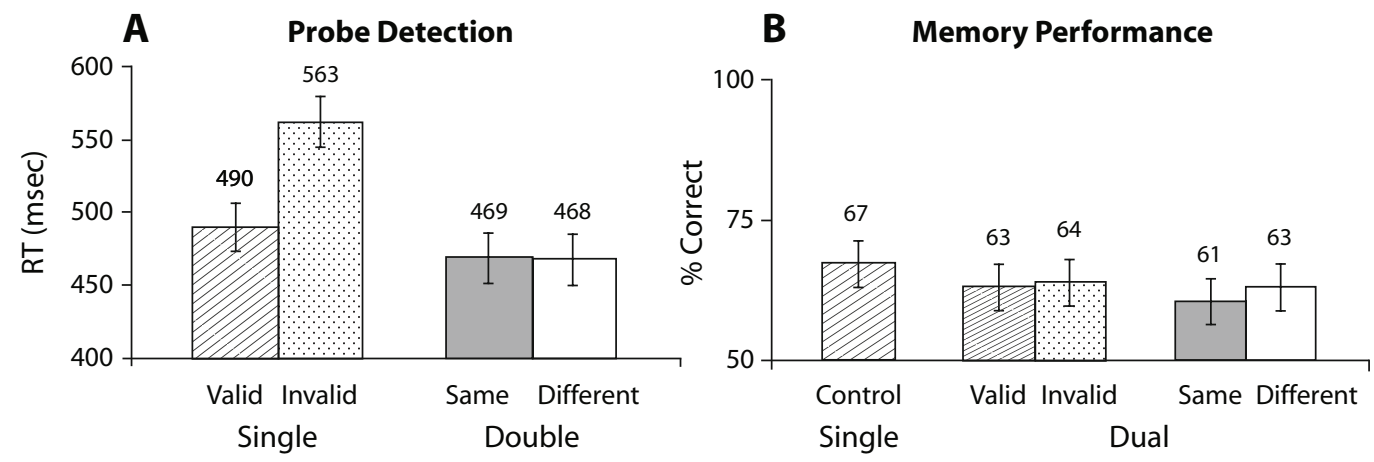

Figure 8. (A) Median response times (RTs) for correct responses for the loaded probe-detection task in Experiment 5. (B) Accuracy of memory performance on the function of task type in Experiment 5. 
Before concluding that observers could use the second cue in the double-cue condition even with a substantial memory load, it is necessary to ensure that performance on the memory task was not traded off against performance on the detection task. Figure 8B illustrates observers' performance for the memory task across two conditions. The shaded bar on the left represents their performance in the change-detection task alone (single-task condition). The remaining four bars represent their performance in both the change-detection and probe-detection tasks (dual-task condition).

Accuracy was well below ceiling in all conditions, suggesting that memory capacity was exceeded by the six to-be-remembered objects. Memory performance in the dual-task condition was approximately $4 \%$ less accurate than that in the single-task condition. There was no significant difference in memory performance between the single-task condition and the dual-task condition $[F(1,9)=2, p>.2]$.

The pattern of these data suggests that observers could use the second cue in the double-cue condition even when their VSTM was occupied. Thus, the failure of observers to show high levels of performance for the differentdirection trials in the double-cue condition of Experiments 3 and 4 does not reflect a general inability or unwillingness to shift attention twice under these timing and probability conditions.

\section{GENERAL DISCUSSION}

These experiments confirm that attention-directing cues can influence the selection of items from VSTM. Griffin and Nobre (2003) reported that cues influenced memory performance even when they appeared long after the iconic image of these objects had faded. This finding seemed mysterious because there was no source of new information that could be selected at the time to improve the representation of the cued items in VSTM. Moreover, this finding appeared to conflict with many previous studies in which postcues were ineffective (Averbach \& Coriell, 1961; Sperling, 1960). The present results indicate that postcues can be used to protect already-formed VSTM representations from becoming degraded. Specifically, once attention has been directed to a location during the retention interval, a cue directing attention to a different location does not lead to improved performance at the new location. Thus, the cue does not act to improve the quality at the cued location, which makes sense, given that there is no source of new information to be used for this purpose. However, in a more standard cuing paradigm (Experiment 5), in which the target information was presented after the cue, a second cue led to improved performance at the location indicated by this cue, showing that observers have the ability to shift attention twice. These results indicate that a cue presented during the retention interval of a change-detection task operates by avoiding the loss of already-acquired information, rather than by the acquisition of new information.

Landman et al. (2003) used a similar double-cuing procedure to examine the capacity of VSTM, but they arrived at a very different result from ours. Specifically, Landman et al. reported equivalent performance when the second cue was different from the first cue (i.e., the differentdirection trial). This result suggests that no information is lost from the uncued items when the first cue appears, consistent with a prioritization account. However, there are two possible methodological problems with Landman et al.'s experiment. First, they provided no means of determining whether their observers attended to the location indicated by the first cue on double-cue trials. If the observers had simply ignored the first cue and attended to the second cue - or had ignored both cues - this could explain the lack of a difference between same-direction and different-direction trials. The pattern of results in Experiment 3 of the present study indicates that observers must have attended to the side indicated by the first cue; otherwise, performance could not have been more accurate on same-direction trials than on different-direction trials. Moreover, we observed a significant difference between valid and invalid trials from the single-cue condition, suggesting that observers attended to the location indicated by the first cue they saw. A second problem with the study of Landman et al. is that they used an orientation change-detection task, in which eight oriented lines were presented around an imaginary circle. It is very likely that the observers grouped these lines into a single shape, and change-detection performance was much more accurate than it could normally be expected for an array of 8 items. Consequently, the lack of a difference between the two types of double-cue trials may simply have been a result of a ceiling effect. In contrast, performance was well away from ceiling in the present experiments.

Although the present results provided clear evidence that attention could be used to protect already-formed VSTM representations, it is still necessary to explain why cues were found to be ineffective after the icon had faded in iconic memory experiments (e.g., Averbach \& Coriell, 1961; Sperling, 1960). A very reasonable explanation is that the iconic memory studies encouraged observers to encode the cued items in verbal working memory by using alphanumeric stimuli and by requiring observers to recall these items verbally. Thus, the cues in the classic partial report procedure are presumably used to control a visual-to-verbal recoding process. If the cue appears after the icon has faded, it is too late to perform this recoding, and the cue will be ineffective. By contrast, most changedetection experiments minimize the role of verbal recoding by using difficult-to-verbalize stimuli, a concurrent verbal memory load, or a concurrent articulatory suppression task. Performance therefore depends on visual rather than verbal working memory, and the protection mechanism identified in the present study may be more important for visual than for verbal working memory.

\section{AUTHOR NOTE}

This research was made possible by a Sigma Xi Grants-in-Aid of Research Award to M.M., National Institute of Mental Health (R01 MH63001) to S.J.L., National Science Foundation (BCS 99-10727) and National Institute of Mental Health (MH60636) to S.P.V. We thank Andrew Hollingworth and three anonymous reviewers for their help- 
ful comments, as well as Mikaela Colbert and Holly Olson for their help with data collection of Experiments 1 and 3, and Elsie Braun and Krista Brittain for their help with programming, data collection, and a part of data analysis of Experiment 5. Correspondence concerning this article should be addressed to M. Matsukura or S. P. Vecera, Department of Psychology, University of Iowa, E11 Seashore Hall, Iowa City, IA 52242-1407 (e-mail: michi-matskura@uiowa.edu, sjluck@ucdavis.edu, or shaun-vecera@uiowa.edu).

\section{REFERENCES}

Averbach, E., \& Coriell, A. S. (1961). Short-term memory in vision. Bell System Technical Journal, 40, 309-328.

BADDELEY, A. D. (1986). Working memory. Oxford: Oxford University Press, Clarendon Press.

Becker, M. W., Pashler, H., \& Anstis, S. M. (2000). The role of iconic memory in change detection tasks. Perception, 29, 273-286.

Besner, D., Davies, J., \& Daniels, S. (1981). Reading for meaning: The effects of concurrent articulation. Quarterly Journal of Experimental Psychology, 33A, 415-437.

Bundesen, C. (1990). A theory of visual attention. Psychological Review, 97, 523-547.

Cowan, N. (1997). Attention and memory. New York: Oxford University Press.

Duncan, J., \& Humphreys, G. W. (1989). Visual search and stimulus similarity. Psychological Review, 96, 433-458.

Griffin, I. C., \& Nobre, A. C. (2003). Orienting attention to locations in internal representations. Journal of Cognitive Neuroscience, $\mathbf{1 5}$ 1176-1194.

IRWIN, D. E. (1992). Memory for position and identity across eye movements. Journal of Experimental Psychology: Learning, Memory, \& Cognition, 18, 307-317.

IRWIN, D. E., \& ANDREws, R. V. (1996). Integration and accumulation of information across saccadic eye movements. In T. Inui \& J. L. McClelland (Eds.), Attention and performance XVI: Information integration in perception and communication (pp. 125-155). Cambridge, MA: MIT Press.

JohnSON, D. N., \& YANTIS, S. (1995). Allocating visual attention: Tests of a two-process model. Journal of Experimental Psychology: Human Perception \& Performance, 21, 1376-1390.

Landman, R., Sperkreisse, H., Lamme, V. A. F. (2003). Large capacity storage of integrated objects before change blindness. Vision Research, 43, 149-164.

Loftus, G. R., \& Masson, M. E. J. (1994). Using confidence intervals in within-subject designs. Psychonomic Bulletin \& Review, 1, 476-490.

Luck, S. J., \& Vecera, S. P. (2002). Attention. In H. Pashler (Series Ed.) \& S. Yantis (Volume Ed.), Stevens' Handbook of experimental psychology: Vol. 1. Sensation and perception (3rd ed., pp. 235-286). New York: Wiley.
LucK, S. J., \& Vogel, E. K. (1997). The capacity of visual working memory for features and conjunctions. Nature, 390, 279-281.

Macmillan, N. A., \& Creelman, C. D. (1991). Detection theory: A user's guide. Cambridge: Cambridge University Press.

MurRaY, D. J. (1968). Articulation and acoustic confusability in shortterm memory. Journal of Experimental Psychology, 78, 679-684.

Posner, M. I. (1980). Orienting of attention. Quarterly Journal of Experimental Psychology, 32, 3-25.

Schmidt, B. K., Vogel, E. K., Woodman, G. F., \& Luck, S. J. (2002). Voluntary and involuntary attentional control of visual working memory. Perception \& Psychophysics, 64, 754-763.

Shomstein, S., \& Yantis, S. (2002). Object-based attention: Sensory modulation or priority setting? Perception \& Psychophysics, 64, 41-51.

Shomstein, S., \& Yantis, S. (2004).Configural and contextual prioritization in object-based attention. Psychonomic Bulletin \& Review, 11, 247-253.

SPERLING, G. (1960). The information available in brief visual presentations. Psychological Monographs, 74,1-29.

Vogel, E. K., Woodman, G. F., \& Luck, S. J. (2001). Storage of features, conjunctions, and objects in visual working memory. Journal of Experimental Psychology: Human Perception \& Performance, 27, 92-114.

Vogel, E. K., Woodman, G. F., \& LucK, S. J. (2006). The time course of consolidation in visual working memory. Journal of Experimental Psychology: Human Perception \& Performance, 32, 1436-1451.

WoJCIUlik, E., \& Kanwisher, N. (1999). The generality of parietal involvement in visual attention. Neuron, 23, 747-764.

Woodman, G. F., \& LucK, S. J. (2003). Serial deployment of attention during visual search. Journal of Experimental Psychology: Human Perception \& Performance, 29, 121-138.

Woodman, G. F., Vecera, S. P., \& Luck, S. J. (2003). Perceptual organization influences visual working memory. Psychonomic Bulletin \& Review, 10, 80-87.

\section{NOTES}

1. To rule out possible distortions from response bias, all the data in this study were also analyzed with $d^{\prime}$, a measure of sensitivity based on the signal detection theory (Macmillan \& Creelman, 1991). The analyses of $d^{\prime}$ yielded the same pattern of results as did the analyses of percent correct.

2. A between-experiments analysis revealed that the size of the cuing effect observed in Experiment 2 did not significantly differ from that observed in the 2,000-msec test-duration trials of set size 4 in Experiment $1[F(1,19)=3, p>.1]$.

(Manuscript received August 18, 2006; revision accepted for publication June 4, 2007.) 